adults in selected counties in California, Colorado, Connecticut, Georgia, Maryland, Michigan, Minnesota, Ohio, Oregon, New Mexico, New York, Tennessee, and Utah with a total catchment population of over 27 million people ( $\sim 9 \%$ of the US population). Using this platform, we will retrospectively evaluate four influenza seasons using FluSurv-NET data to look at the timing of influenza vaccination and severity of illness among patients with influenzarelated hospitalization. We will conduct a multivariate analysis to assess for differences in severe outcomes including duration of hospitalization, ICU admission, and death among patients with varying lengths of time between influenza vaccination and influenza-related hospitalization. Separate analyses will be performed among different age groups and influenza type/subtypes, as well as specific seasons as a surrogate for most common circulating strain. RESULTS/ANTICIPATED RESULTS: We hypothesize that patients with chronic medical conditions and those at the extremes of age will have a longer duration between vaccination and hospitalization as they are more likely to get vaccinated earlier. We also hypothesize that patients with longer duration between seasonal influenza vaccination and hospitalization will have a longer duration of hospitalization and a higher rate of other severe outcomes (e.g., ICU admission, death). Such data would suggest that immune protection wanes during the influenza season. DISCUSSION/ SIGNIFICANCE OF IMPACT: Limited data suggest that vaccineinduced influenza immunity may wane during the influenza season. It is not known whether the impact of influenza vaccination upon severity of disease might wane with increasing time between vaccination and influenza infection. In contrast to many previous studies evaluating vaccine effectiveness which have assessed medically-attended influenza illness as a primary outcome, our dataset is a large cohort of hospitalized patients which allows us to assess rare yet critical outcomes such as ICU admission and death. This study will also have a substantially larger amount of pediatric data than previous studies, which will provide the opportunity to determine whether timing of vaccination affects children and adults differently. Improving our understanding of whether influenza vaccine-induced protection might wane over time could ultimately impact U.S. influenza vaccination policy resulting in decreased morbidity and mortality attributed to influenza each season.

\section{Torsade de pointes/QT prolongation risks with antibiotics: A contemporary analysis of the FDA Adverse Event Reporting System}

Chengwen Teng ${ }^{1}$, Daryl Kevin S. Gaspar and Christopher Frei

${ }^{1}$ University of Texas Health Science Center San Antonio

OBJECTIVES/SPECIFIC AIMS: Macrolides, linezolid, imipenemcilastatin, fluoroquinolones, penicillin combinations, and ceftriaxone are known to be associated with Torsade de pointes/QT prolongation (TdP/QTP). Other antibiotics may also lead to TdP/QTP, but no study has systemically compared TdP/QTP risks of different antibiotics using recent data. Therefore, the objective of this study was to evaluate the association between TdP/QTP and antibiotics in recent years using the FDA Adverse Event Report System (FAERS). METHODS/STUDY POPULATION: FAERS reports from January 1, 2015 to December 31, 2017 were analyzed. The Medical Dictionary for Regulatory Activities (MedDRA) was used to identify TdP/QTP cases. We calculated the Reporting Odds Ratios (RORs) and corresponding 95\% confidence intervals (95\%CI) for the association between antibiotics and TdP/QTP. An association was considered to be statistically significant when the lower limit of the $95 \% \mathrm{CI}$ was greater than 1. RESULTS/ANTICIPATED RESULTS: A total of 2,042,801 reports (including 5,221 TdP/QTP reports) were considered, after inclusion criteria were applied. Macrolides had the greatest proportion of TdP/QTP reports, representing $2.9 \%$ of all macrolide reports. TdP/QTP RORs (95\%CI) for the antibiotics were (in descending order): macrolides 11.73 (9.74-14.12), linezolid 9.39 (6.45-13.68), amikacin 8.94 (4.22-18.92), imipenem-cilastatin 5.01 (2.38-10.56), fluoroquinolones 4.67 (3.96-5.52), penicillin combinations 3.52 (2.56-4.86), cephalosporins 1.90 (1.14-3.16), metronidazole 1.49 (0.74-2.99), vancomycin 1.26 (0.70-2.28), clindamycin 0.83 (0.27-2.58), trimethoprim-sulfamethoxazole 0.82 (0.31-2.18), and amoxicillin $0.57(0.18-1.78)$. DISCUSSION/SIGNIFICANCE OF IMPACT: This study confirms prior evidence for TdP-QTP risks with macrolides, linezolid, imipenem-cilastatin, fluoroquinolones, penicillin combinations, and cephalosporins. This study provides new evidence for TdP-QTP risks with amikacin. Macrolides had the highest TdP/QTP ROR among the antibiotics evaluated in this study.

3317

Translating simulation-based team leadership training into patient-centered outcomes

Rosemarie Fernandez ${ }^{1}$, Elizabeth D. Rosenman, Anne K. Chipman, Sarah Brolliar, Marie C. Vrablik, Anthony T Misisco, Jeff Olenick, James Grand, Colleen Kalynych, Steve W. J. Kozlowski and

Georgia T. Chao

${ }^{1}$ University of Florida Clinical and Translational Science Institute

OBJECTIVES/SPECIFIC AIMS: The objective of this research was to assess the clinical impact of simulation-based team leadership training on team leadership effectiveness and patient care during actual trauma resuscitations. This translational work addresses an important gap in simulation research and medical education research. METHODS/STUDY POPULATION: Eligible trauma team leaders were randomized to the intervention (4-hour simulation-based leadership training) or control (standard training) condition. Subject-led actual trauma patient resuscitations were video recorded and coded for leadership behaviors (primary outcome) and patient care (secondary outcome) using novel leadership and trauma patient care metrics. Patient outcomes for trauma resuscitations were obtained through the Harborview Medical Center Trauma Registry and analyzed descriptively. A one-way ANCOVA analysis was conducted to test the effectiveness of our training intervention versus a control group for each outcome (leadership effectiveness and patient care) while accounting for pre-training performance, injury severity score, postgraduate training year, and days since training occurred. Association between leadership effectiveness and patient care was evaluated using random coefficient modeling. RESULTS/ ANTICIPATED RESULTS: Sixty team leaders, 30 in each condition, completed the study. There was a significant difference in posttraining leadership effectiveness $[\mathrm{F}(1,54)=30.19, \mathrm{p}<.001, \eta 2=.36]$ between the experimental and control conditions. There was no direct impact of training on patient care $[\mathrm{F}(1,54)=1.0, \mathrm{p}=0.33$, $\eta 2=.02$ ]; however, leadership effectiveness mediated an indirect effect of training on patient care. Across all trauma resuscitations 